\title{
Análise dos Efeitos da Dupla Tarefa na Marcha de Pacientes com Doença de Parkinson: Relato de Três Casos
}

\author{
Analysis of the Effects of Dual Task in Gait of Parkinson's Disease Patients: Three Cases Report
}

\author{
Daiane Pishinin Cândido', Bianca A. de Luca Cillo², Alexandre Saeki \\ Fernandes ${ }^{3}$, Rosana Pazim Nalesso ${ }^{4}$, Fabio Jakaitis ${ }^{5}$, Daniel Gonçalves dos \\ Santos $^{6}$
}

\section{RESUMO}

Introduçáo. A doença de Parkinson (DP) apresenta como um dos sintomas mais incapacitantes a marcha, que pode ser denominada de festinação ou petit pass junto com o bloqueio motor. A dupla tarefa (DT) vem sendo utilizada como recurso terapêutico vantajoso e um pré-requisito no desempenho funcional nas diversas atividades de vida diária. Objetivo. Analisar a influência da dupla tarefa na velocidade da marcha e equilíbrio de pacientes com DP. Método. Estudo de caso com 3 pacientes do centro clínico Uniban $\mathrm{ABC}$, com DP, ambos os sexos e idades entre 56 e 76 anos, com alteração da marcha. Os dados foram analisados antes e após o treinamento, através da escala de equilíbrio de Berg e do teste Time up and Go (TUG). O treinamento teve duração de três meses, duas vezes por semana, totalizando dezesseis sessôes. Resultados. Os resultados mostraram que a tarefa cognitiva não influenciou o desempenho motor da marcha. Porém, promoveu melhora no equilíbrio e na mobilidade funcional. Conclusáo. Verificou-se que o treinamento em condição de dupla tarefa cognitivo/motora pode ser eficaz no desempenho da marcha de pacientes com DP.

Unitermos. Doença de Parkinson, Marcha, Cognição.

Citaçáo. Cândido DP, Cillo BAL, Fernandes AS, Nalesso RP, Jakaitis F, Santos DG. Análise dos Efeitos da Dupla Tarefa na Marcha de Pacientes com Doença de Parkinson: Relato de Três Casos.

\begin{abstract}
Introduction. Parkinson's disease (PD) presents as one of the most disability symptoms the gait, denominated festination or petit pass, along with freezing. The dual task (DT) has been used as a therapeutic resource. Advantageous and a prerequisite in functional performance in daily life activity. Objective. Analyze the influence of dual task in gait speed and balance of PD patients. Method. Case study with 3 patients of UNIBAN clinical center with PD, both sexes and aged between 56 and 76 years, with gait's change. The data were analyzed before and after training, by Berg Balance Scale and Time Up and Go test. The training was realized thought three months, twice a week, total 16 sessions. Results. The results showed that cognitive task did not influence the gait motor performance. However, it raised improvement in balance and functional mobility. Conclusion. It was observed that dual task cognitive/motor condition training can be effective in gait performance of PD patients.
\end{abstract}

Keywords. Parkinson's disease, Gait, Cognitive.

Citation. Cândido DP, Cillo BAL, Fernandes AS, Nalesso RP, Jakaitis F, Santos DG. Analysis of the Effects of Dual Task in Gait of Parkinson's Disease Patients: Three Cases Report.

\footnotetext{
Trabalho realizado na Universidade Bandeirante de Sáo Paulo (UNIBAN), campus ABC, São Bernardo do Campo-SP, Brasil.

1. Fisioterapeuta Universidade Bandeirante de São Paulo (UNIBAN), São Bernardo do Campo-SP, Brasil.

2. Fisioterapeuta, Mestre USP Professora e supervisora do estágio de fisioterapia em neurologia da UNIBAN, São Bernardo do Campo-SP, Brasil.

3. Fisioterapeuta Especialista em Fisioterapia motora Professor e Supervisor do estágio de ortopedia e traumatologia da UNIBAN, São Bernardo do CampoSP, Brasil.

4. Fisioterapeuta, Mestre Professora Titular da Universidade Bandeirante de São Paulo (UNIBAN), Sáo Bernardo do Campo-SP, Brasil.

5. Fisioterapeuta Especialista em Fisiologia do Exercício, Professor Titular da Universidade Bandeirante de São Paulo (UNIBAN), São Bernardo do CampoSP, Brasil.

6. Fisioterapeuta, Especialista, Hospital Israelita Albert Einstein, São Paulo-SP, Brasil.
}

Endereço para correspondência: Bianca A de Luca Cillo Av. Dr. Rudge Ramos, 1.501 CEP 09636-000, São Bernardo do Campo-SP, Brasil. E-mail: bia.luca@uol.com.br

Relato de Caso Recebido em: 31/01/11 Aceito em: 24/08/11 Conflito de interesses: não 


\section{INTRODUÇÃO}

A DP está entre as principais doenças neurodegenerativas do SNC, ocasionada pela diminuição na produção do neurotransmissor dopamina, devido à degeneração dos neurônios dopaminérgicos que se encontram na parte compacta da substância negra um dos núcleos da base, situada no mesencéfalo ${ }^{1}$.

Com prevalência estimada em 1 a $2 \%$ da população mundial, acomete preferencialmente o sexo masculino acima de $60 \mathrm{anos}^{2}$. Seu início ocorre de forma insidiosa afetando primeiramente um lado do corpo, tornando-se bilateral com a progressão da doença ${ }^{3}$. O quadro clínico é caracterizado principalmente por sintomas motores, entre os quais se destacam a bradicinesia, a rigidez muscular, o tremor de repouso, as alteraçôes da postura e da marcha ${ }^{4}$.

A marcha é um dos sintomas mais incapacitantes na DP, podendo ser denominada de festinação ou petit pass, é caracterizada pela pobreza dos movimentos, passos curtos, pés rentes ao chão e diminuição da velocidade. Com a progressão da doença ocorre um aumento progressivo na velocidade e no número de passos para atingir o centro de gravidade, na tentativa de evitar as quedas ${ }^{2,3}$.

Outra característica apresentada durante a marcha é o bloqueio motor também conhecido como freezing, que costuma ocorrer quando o paciente inicia a marcha ou quando tenta fazer uma volta e até mesmo por hesitação ao passar por barreiras, como portas giratórias, corredores estreitos, atravessar ruas movimentadas. Por este motivo a marcha é o objeto de estudo de diversos trabalhos visando estratégias na sua reabilitação ${ }^{5}$.

A DT, também conhecida como tarefa simultânea ou tarefa associada, é aprendida durante toda a vida. Altamente vantajosa, é um pré-requisito no desempenho funcional nas diversas atividades de vida diária. Uma caminhada, por exemplo, permite a comunicação entre pessoas, atender um celular, transportar objeto de um local para outro, entre outras. Em circunstâncias normais a realização simultânea das tarefas cognitiva e motora é desempenhada automaticamente, enquanto, quando se apresenta uma alteração cognitiva e/ou no controle motor requer uma demanda atencional maior, podendo comprometer o desempenho em ambas. Estudos apontam a possibilidade de melhora da marcha e do equilíbrio, com o treinamento da DT no processo de reabilitação em indivíduos com $\mathrm{DP}^{6,7}$.

As pistas visuais são utilizadas para desviar a função dos núcleos da base a fim de regular a função motora deficitária. Essas pistas têm acesso a mecanismos de controle motor, envolvidos no aprendizado e recrutamento de sinais adicionais, que levam a um desvio da preparação do movimento do circuito núcleos da base - área motora suplementar, para a área visual-motora, cerebelo e córtex pré-motor. Melhorando a preparação do passo, funcionam alternativamente por focarem a atenção do paciente para sua execução ${ }^{8}$.

O presente estudo teve como objetivo analisar a influência da DT na velocidade da marcha e equilíbrio de pacientes com DP.

\section{MÉTODO}

\section{Relato de três casos}

A pesquisa foi realizada no Centro Clínico UNIBAN ABC após os pacientes assinarem os termos de consentimento livre e esclarecido.

Foram avaliados três indivíduos, no qual estiveram de acordo com os critérios de inclusão: DP diagnosticada pelo neurologista, idades entre 56 e 76 anos, ambos os sexos, Mini-exame do estado mental (MEEM) maior que 24 pontos (nota de corte utilizada 18), Hoehn e Yahr (modificada) com escore entre 2,5 e 3, Índice de Barthel maior que 10 e escala de equilíbrio de Berg maior que 36 pontos, também foi utilizada a UPDRS (Escala Unificada de Avaliação da DP) visando determinar os critérios de inclusão e exclusão, assim como as características clínicas dos pacientes (Quadro 1). Já a escala de equilíbrio de Berg e o teste TUG foram aplicados pré e pós treinamento para obtenção dos resultados.

A Escala de equilíbrio de Berg foi desenvolvida em 1992 por Katherine Berg para avaliar o risco de quedas em idosos, sendo traduzida e adaptada para a língua portuguesa em 2004 por Miyamoto et al. em sua dissertação de mestrado. Essa escala avalia o equilíbrio estático e dinâmico envolvendo 14 tarefas comuns da vida diária como alcançar, girar, transferir-se, levantar-se e permanecer em pé. A avaliação é feita através de observação e supervisão se necessário para execução da tarefa. A pontuação varia de 0 a 4 em uma escala totalizando 56 pontos. A pontua- 
Quadro 1

Características dos pacientes

\begin{tabular}{|c|c|c|c|}
\hline & Paciente 1 & Paciente 2 & Paciente 3 \\
\hline Sexo e idade & Masculino, 56 anos & Feminino, 68 anos & Feminino, 75 anos \\
\hline Escolaridade & $\begin{array}{l}\text { Ensino fundamental } \\
\text { incompleto }\end{array}$ & Ensino Superior & Não alfabetizada \\
\hline $\begin{array}{l}\text { Mini-exame do } \\
\text { estado mental }\end{array}$ & Pontuação 27 & Pontuação 27 & Pontuação 18 \\
\hline $\begin{array}{l}\text { Diagnóstico da DP } \\
\text { (em anos) }\end{array}$ & 5 anos & 7 anos & 8 anos \\
\hline Medicamentos & $\begin{array}{l}\text { Prolopa, Mantidan, } \\
\text { Captopril }\end{array}$ & Prolopa, Akineton & Mantidan, Parken, Sifrol \\
\hline $\begin{array}{l}\text { Hoehn e Yahr } \\
\text { (Modificada) }\end{array}$ & Pontuação 2,5 & Pontuação 3 & Pontuação 2,5 \\
\hline Índice de Barthel & Pontuação 16 & Pontuação 19 & Pontuação 18 \\
\hline $\begin{array}{l}\text { Principal } \\
\text { característica clínica }\end{array}$ & $\begin{array}{l}\text { Tremor de repouso em } \\
\text { membros superiores bila- } \\
\text { teral com padráo flexor }\end{array}$ & $\begin{array}{l}\text { Marcha festinada e } \\
\text { bloqueio motor }\end{array}$ & $\begin{array}{l}\text { Hipertonia plástica e } \\
\text { postura cifótica }\end{array}$ \\
\hline $\begin{array}{l}\text { Marcha de acordo } \\
\text { com a UPDRS }\end{array}$ & $\begin{array}{l}\text { Pontuaçáo } 1 \text { (anda lenta- } \\
\text { mente, pode arrastar os } \\
\text { pés com pequenas passa- } \\
\text { das, mas não há festinação } \\
\text { ou propulsão) }\end{array}$ & $\begin{array}{l}\text { Pontuação } 2 \text { (anda com } \\
\text { dificuldade, mas precisa } \\
\text { de pouca ajuda ou ne- } \\
\text { nhuma, pode apresentar } \\
\text { alguma festinação, passos } \\
\text { curtos, ou propulsão) }\end{array}$ & $\begin{array}{l}\text { Pontuação } 1 \text { (anda lenta- } \\
\text { mente, pode arrastar os } \\
\text { pés com pequenas passa- } \\
\text { das, mas não há festinaçãa } \\
\text { ou propulsão) }\end{array}$ \\
\hline
\end{tabular}

ção abaixo de 36 pontos indica $100 \%$ de risco de quedas 9

O TUG é um teste cronometrado onde se mensura o tempo em que o indivíduo leva para levantar de uma cadeira, caminhar 3 metros em velocidade habitual com seus calçados usuais, virar-se e retornar sentando novamente na cadeira. Este teste tem como finalidade avaliar a mobilidade funcional, o nível de equilíbrio e predizer o risco de quedas. A pontuação até 10 segundos é normal em indivíduos saudáveis, de 11 a 20 segundos são considerados dentro dos limites normais para idosos frágeis ou indivíduos com incapacidade e acima de 20 segundos são indicativos de mobilidade funcional comprometida com alto risco de quedas ${ }^{2,10}$.

\section{Procedimentos}

A intervençáo foi composta por uma tarefa motora que consistiu em treino de marcha realizado em uma pista visual/obstáculos e uma tarefa cognitiva composta por 3 slides com sequências de formas e cores, realizados em um local sem interferências externas (Figura 1).

O treino de marcha foi realizado em um trajeto de 5 metros, contendo 4 obstáculos posicionados a 10 centímetros do chão e a uma distância de 1 metro entre eles, intercalados nas cores vermelha e verde. Os pacientes eram orientados para que realizassem a tarefa motora iniciando o $1^{\circ}$ obstáculo (cor vermelha) com o membro inferior direito e o $2^{\circ}$ obstáculo (cor verde) com o membro inferior esquerdo e assim sucessivamente. Ao chegar ao último sarrafo de cor verde, fizessem a volta e retornassem, respeitando as passadas com o membro correspondente a cor.

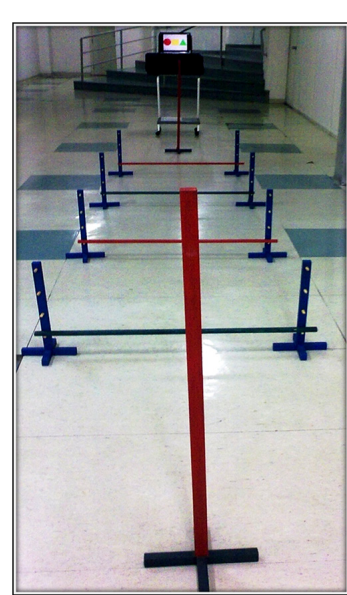

Slide 1

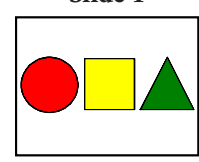

Slide 2

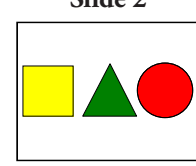

Slide 3

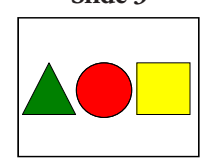

Figura 1. Tarefa motora e tarefa cognitiva. 
A tarefa cognitiva consistiu de uma apresentação de slides com três sequências contendo 3 formas geométricas simples preenchidas com cores (vermelho, amarelo e verde), apresentada na tela do computador padronizado com medidas: 30 × 20 centímetros que estava a 1 metro de distância de um dos sarrafos sob um suporte de 1 metro de altura. As tarefas foram realizadas isoladamente na primeira intervençáo para que houvesse o reconhecimento da tarefa proposta pelos pacientes.

Após o reconhecimento das tarefas motora e cognitiva, os pacientes realizaram o treinamento simultâneo de ambas, iniciando pela marcha e ao retornar passando pelo primeiro obstáculo era exposto na tela o primeiro slide que permanecia por 20 segundos, o paciente retornava e então descrevia respectivamente a sequência de formas e cores. Cada paciente realizava o percurso 3 vezes até cessarem os três slides, retornando a posição inicial.

\section{RESULTADOS}

Após o treinamento, os pacientes apresentaram alteraçóes no equilíbrio estático e dinâmico. $\mathrm{Na}$ escala de equilíbrio de Berg, o paciente 1 apresentou 48 e 50 pontos na avaliação inicial e final, respectivamente obtendo assim 2 pontos de melhora no equilíbrio. Este resultado também pode ser visto na paciente 2 que iniciou com 47 pontos e finalizou o treinamento com 49 pontos. Já a paciente 3 iniciou com 45 pontos e finalizou com 50 (Figura 2).

Referente ao TUG nota-se através dos dados obtidos na Tabela 1, uma redução do tempo gasto para realizar o teste em 1 segundo após o treinamento. O paciente

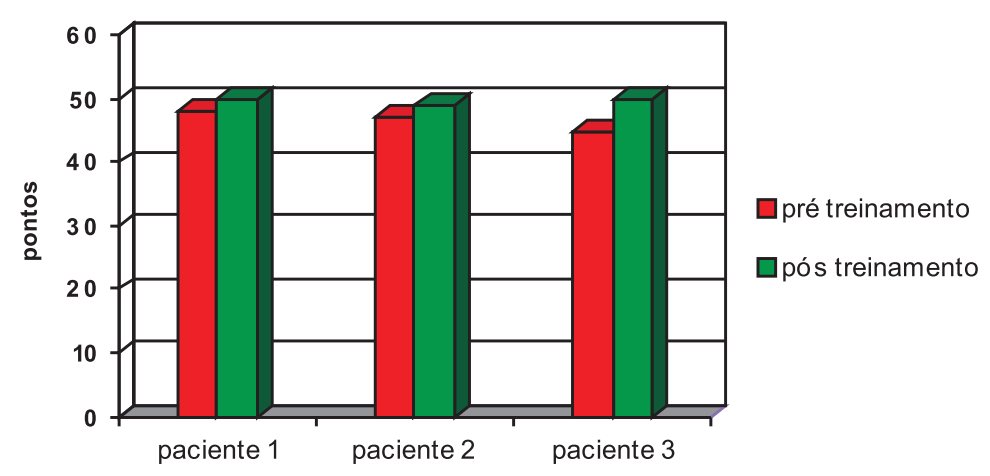

Figura 2. Resultados da Escala de Equilíbrio de Berg pré e pós treinamento.
1 realizou o teste pré treinamento em 19 segundos, e após o treinamento o tempo foi de 18 segundos. A paciente 2 teve seu tempo de 21 segundos reduzido para 20 segundos após o treinamento e a paciente 3 apresentou a redução do seu tempo de 14 segundos para 13 segundos.

\section{DISCUSSÃO}

A DP é uma patologia caracterizada principalmente por sintomas motores, dentre as quais, a marcha é sem dúvida a mais estudada pelo seu grande impacto funcional nas AVD's dos pacientes ${ }^{11}$. Em busca de melhores condutas fisioterapêuticas, diversos trabalhos têm utilizado estímulos visuais durante o treino de marcha com o intuito de tentar corrigir as alteraçóes provocadas pela patologia ${ }^{8,12}$.

Os indivíduos com DP têm mais facilidade em subir degraus do que andar em uma superfície plana, isso ocorre devido à estimulação visual, que também é benéfica contra episódios de bloqueio motor ${ }^{4}$. O bloqueio motor pode surgir quando o paciente se depara com um obstáculo real ou apenas visual, como uma pequena elevação ou uma faixa pintada no solo ${ }^{13}$. Por isto, foram dadas preferências às pistas visuais (obstáculos), por estarem presentes no nosso dia a dia tanto no ambiente interno quanto externo, como escadas, calçadas irregulares e degraus.

Sabe-se que, pacientes com DP possuem maior dificuldade na realização das tarefas simultâneas comparada com tarefas simples e por isso devem completar um movimento antes de começar a realizar o próximo e que o treinamento em condiçẫo de tarefa única é gerenciado por áreas diferentes de uma DT, o que leva a uma maior ativação decorrente da aquisição de uma nova capacidade de dividir a atenção, o que não acontece quando se treina uma única tarefa ${ }^{3,7}$. Por ser frequente a condição de DT nas atividades de vida diárias, devem ser incluídas no processo de reabilitação, visando a qualidade de vida dos pacientes ${ }^{7}$. A DT cognitivo/motora foi escolhida para a realização deste estudo por proporcionar um gerenciamento de divisão de atenção que está comprometido na DP, principalmente durante a marcha. 
Tabela 1

Descrição dos valores em segundos avaliados pelo Teste Time Up and Go

\begin{tabular}{cccc}
$\begin{array}{c}\text { Teste Time up } \\
\text { and Go }\end{array}$ & $\begin{array}{c}\text { Pré treinamento } \\
\text { (segundos) }\end{array}$ & $\begin{array}{c}\text { Pós treinamento } \\
\text { (segundos) }\end{array}$ & $\begin{array}{c}\text { Ganho } \\
\text { (segundos) }\end{array}$ \\
\hline Paciente 1 & 19 & 18 & $\mathbf{1}$ \\
Paciente 2 & 21 & 20 & $\mathbf{1}$ \\
Paciente 3 & 14 & 13 & $\mathbf{1}$ \\
\hline
\end{tabular}

Nas atividades diárias como atravessar a rua, levar o cão para passear, fazer compras, falar ao celular enquanto caminha e até mesmo dirigir exige um gerenciamento simultâneo dos componentes visual e motor. Esse aprendizado ocorre durante toda a vida e pode ser comprometido pela idade, escolaridade, experiência prévia e principalmente pelas patologias ${ }^{7}$. No presente estudo foram encontradas essas associaçóes principalmente na paciente 3 o que pode ter contribuído para redução dos resultados apontados em comparaçáo com os outros dois pacientes.

O teste Time Up and Go tem como finalidade avaliar a mobilidade funcional, o nível de equilíbrio e predizer o risco de quedas, onde a pontuação até 10 segundos é normal em indivíduos saudáveis, de 11 a 20 segundos são considerados dentro dos limites normais para idosos frágeis ou indivíduos com incapacidade e acima de 20 segundos são indicativos de mobilidade funcional comprometida $^{2,10}$. Segundo esse padrão, os pacientes 1 e 3 apresentaram o tempo dentro dos limites normais para o teste. A paciente 2 apresentou um valor inicial de 21 segundos para realizar o teste, o que representa indícios de mobilidade funcional comprometida ${ }^{2,5}$.

A diminuição do tempo gasto para realizar o teste como apontados nos resultados é considerado benéfico, uma vez que as características da marcha são passos cada vez mais curtos ocorrendo à diminuição da velocidade, podendo considerar também um número menor de bloqueios motores durante a marcha.

Em um estudo que foi realizado o treino de marcha em solo estável com pistas visuais associadas a uma tarefa motora, no total de 20 sessões de fisioterapia, houve uma melhora no equilíbrio (analisados pela escala de equilíbrio de Berg) ${ }^{8}$. No presente trabalho também foram constatados resultados relevantes em 16 sessóes, efeitos esses já suficientes para a melhora do equilíbrio com a utilização da DT cognitivo/motora.
Um estudo comparou a introdução de tarefas cognitivas e motoras durante o treino de marcha, e demonstrou que a imposição de tarefas cognitivas era prejudicial à marcha, enquanto as tarefas motoras não surtiam efeitos positivos nem negativos ${ }^{14}$. Já em outro estudo ficou comprovado que as tarefas motoras surtiram efeitos positivos na marcha de pacientes com $\mathrm{DP}^{8}$. Neste estudo os resultados sugerem que a tarefa cognitiva não prejudicou o treino de marcha quando realizados em condição de DT.

\section{CONCLUSÃO}

Com base na interpretaçáo dos resultados obtidos no presente estudo foi possível concluir que o treino em condição de DT cognitivo/motora não interferiu no desempenho motor da marcha dos pacientes. Porém promoveu uma melhora no equilíbrio estático e dinâmico e na mobilidade funcional.

Para melhores resultados, sugere-se a prática desta DT cognitivo/motora por um maior tempo, com uma maior amostra e com diferentes tarefas - motora/motora, visual/motora que enfatizem as dificuldades apresentadas com a progressão das características, as quais afetam de forma negativa a qualidade de vida do paciente com DP.

\section{REFERÊNCIAS}

1.Fahn Stanley, Przedborski Serge. Parkinsonismo. In: Rowland, Lewis P. Merritt: tratado de neurologia. 10. ed. Rio de Janeiro: Guanabara Koogan, 2002, p. 589-602.

2.O' Sullivan SB. Doença de Parkinson. In: O' Sullivan SB, Schimitd TJ. Fisioterapia: avaliação e tratamento. Barueri: Manole;2004, p.747-82.

3.Umphred DA. Reabilitação neurológica. 4 ed. Barueri: Manole, 2004, $1118 \mathrm{p}$.

4.Okamoto E. Comparação entre os efeitos do treinamento motor isolado e do treinamento motor em condição de dupla tarefa, sobre a marcha e a atenção, em idosos saudáveis e pacientes com doença de Parkinson [Dissertação]. São 
Paulo: Universidade de São Paulo, 2008, 122p.

5.Almeida QJ, Lebold CA. Freezing of gait in Parkinson. J Neurol Neurosur Psychiat 2010;81:513-8.

http://dx.doi.org/10.1136/jnnp.2008.160580

6. Teixeira NB, Alouche SR. O desempenho da dupla tarefa na doença de Parkinson. Rev Bras Fisioter 2007;11:127-32.

http://dx.doi.org/10.1590/S1413-35552007000200007

7.Voos MC, Pinheiro GB, Cicca LO, Lázaro A, Valle LER, Piemonte MEP.

Os componentes motor e visual de uma tarefa-dupla devem ser associados ou isolados durante o treinamento? Fisioter Pesq 2008;15:33-9.

8.Dias NP, Fraga DA, Cacho EWA, Oberg TD. Treino de marcha com pistas visuais no paciente com doença de Parkinson. Fisioter Mov 2005;18:43-51.

9.Miyamoto ST, Lombardi Junior I, Berg KO, Ramos LR, Natour J. Brazilian version of the Berg balance scale. Braz J Med Biol Res [serial on the Internet] 2004;37:1411-21.

http://dx.doi.org/10.1590/S0100-879X2004000900017
10.Guimarães LHCT, Galdino DCA, Martins FLM, Vitorino DFM, Pereira KL, Carvalho EM. Comparação da propensão de quedas entre idosos que praticam atividade física e idosos sedentários. Rev Neurocienc 2004;12:68-72.

11.Limongi JCP. Conhecendo melhor a doença de Parkinson: uma abordagem multidisciplinar com orientaçôes práticas para o dia-a-dia. São Paulo: Plexus, 2001, 165p.

12.Sousa CB. Efeitos de um treinamento em condiçấo de dupla-tarefa sobre o desempenho motor e a habilidade de dividir a atençăo em pacientes com doença de Parkinson [dissertação]. São Paulo: Universidade de São Paulo; 2008, 180p. 13. Carvalho Filho ET, Netto MP. Geriatria: fundamentos, clínica e terapêutica. São Paulo: Atheneu, 2004, 447p.

14.Rochester L, Hetherington V, Jones D, Nieuwboer A, Willems A-M, Kwakkel G, et al. Attending to the task: interference effects of funcional tasks on walking in Parkinson's disease and the roles of cognition, depression, fatigue, and balance. Arch Phys Med Rehabil 2004;85:1578-85.

http://dx.doi.org/10.1016/j.apmr.2004.01.025 